\title{
A NEW EXTENSION OF EXTENDED CAPUTO FRACTIONAL DERIVATIVE OPERATOR
}

\author{
GAUHAR RAHMAN, KOTTAKKARAN SOOPPY NISAR*, SHAHID MUBEEN
}

\begin{abstract}
Recently, different extensions of the fractional derivative operator are found in many research papers. The main aim of this paper is to establish an extension of the extended Caputo fractional derivative operator. The extension of an extended fractional derivative of some elementary functions derives by considering an extension of beta function which includes the Mittag-Leffler function in the kernel. Further, an extended fractional derivative of some familiar special functions, the Mellin transforms of newly defined Caputo fractional derivative operator and the generating relations for extension of extended hypergeometric functions also presented in this study.
\end{abstract}

\section{INTRODUCTION}

The Gauss hypergeometric function is defined (see [19]) as

$$
\begin{array}{r}
{ }_{2} F_{1}\left(\vartheta_{1}, \vartheta_{2} ; \vartheta_{3} ; z\right)=\sum_{n=0}^{\infty} \frac{\left(\vartheta_{1}\right)_{n}\left(\vartheta_{2}\right)_{n}}{\left(\vartheta_{3}\right)_{n}} \frac{z^{n}}{n !},(|z|<1), \\
\left(\vartheta_{1}, \vartheta_{2}, \vartheta_{3} \in \mathbb{C} \operatorname{Cand} \vartheta_{3} \neq 0,-1,-2,-3, \cdots\right),
\end{array}
$$

and the integral representation of (1.1) is

$$
{ }_{2} F_{1}\left(\vartheta_{1}, \vartheta_{2} ; \vartheta_{3} ; z\right)=\frac{\Gamma\left(\vartheta_{3}\right)}{\Gamma\left(\vartheta_{2}\right) \Gamma\left(\vartheta_{3}-\vartheta_{2}\right)} \int_{0}^{1} t^{\vartheta_{2}-1}(1-t)^{\vartheta_{3}-\vartheta_{2}-1}(1-z t)^{-\vartheta_{1}} d t
$$

where $\Re\left(\vartheta_{3}\right)>\Re\left(\vartheta_{2}\right)>0$ and $|\arg (1-z)|<\pi$.

The Appell series or bivariate hypergeometric series defined by

$$
F_{1}\left(\vartheta_{1}, \vartheta_{2}, \vartheta_{3} ; \vartheta_{4} ; x, y\right)=\sum_{m, n=0}^{\infty} \frac{\left(\vartheta_{1}\right)_{m+n}\left(\vartheta_{2}\right)_{m}\left(\vartheta_{3}\right)_{n} x^{m} y^{n}}{\left(\vartheta_{4}\right)_{m+n} m ! n !}
$$

for all $\vartheta_{1}, \vartheta_{2}, \vartheta_{3}, \vartheta_{4} \in \mathbb{C}, \vartheta_{4} \neq 0,-1,-2,-3, \cdots, \quad|x|<1,|y|<1$, and the integral representation of (1.3) is given by

$$
F_{1}\left(\vartheta_{1}, \vartheta_{2}, \vartheta_{3}, \vartheta_{4} ; x, y\right)=\frac{\Gamma\left(\vartheta_{4}\right)}{\Gamma\left(\vartheta_{1}\right) \Gamma\left(\vartheta_{4}-\vartheta_{1}\right)}
$$

2010 Mathematics Subject Classification. 26A33,33C20, 33C15, 33C05, 33C65.

Key words and phrases. Hypergeometric function, beta function, Extended hypergeometric function, Mellin transform, fractional derivative, Caputo fractional derivative, Appell's function, generating relation.

*Corresponding author. 


$$
\times \int_{0}^{1} t^{\vartheta_{1}-1}(1-t)^{\vartheta_{4}-\vartheta_{1}-1}(1-x t)^{-\vartheta_{2}}(1-y t)^{-\vartheta_{3}} d t
$$

where $\Re\left(\vartheta_{4}\right)>\Re\left(\vartheta_{1}\right)>0,|\arg (1-x)|<\pi$ and $|\arg (1-y)|<\pi$.

The extended beta function is given in [2] as

$$
B\left(\vartheta_{1}, \vartheta_{2} ; p\right)=B_{p}\left(\vartheta_{1}, \vartheta_{2}\right)=\int_{0}^{1} t^{\vartheta_{1}-1}(1-t)^{\vartheta_{2}-1} e^{-\frac{p}{t(1-t)}} d t
$$

$\left(\Re(p)>0, \Re\left(\vartheta_{1}\right)>0, \Re\left(\vartheta_{2}\right)>0\right)$ respectively. When $p=0$, then $\beta\left(\vartheta_{1}, \vartheta_{2} ; 0\right)=\beta\left(\vartheta_{1}, \vartheta_{2}\right)$, where $\beta\left(\vartheta_{1}, \vartheta_{2}\right)$ is the classical beta function which is defined by

$$
B\left(\vartheta_{1}, \vartheta_{2}\right)=\int_{0}^{1} t^{\vartheta_{1}-1}(1-t)^{\vartheta_{2}-1} d t,\left(\Re\left(\vartheta_{1}\right)>0, \Re\left(\vartheta_{2}\right)>0\right)
$$

The extended hypergeometric and confluent hypergeometric functions is given in [3] as follows:

$$
F_{p}\left(\vartheta_{1}, \vartheta_{2} ; \vartheta_{3} ; z\right)=\sum_{n=0}^{\infty} \frac{B_{p}\left(\vartheta_{2}+n, \vartheta_{3}-\vartheta_{2}\right)}{B\left(\vartheta_{2}, \vartheta_{3}-\vartheta_{2}\right)}\left(\vartheta_{1}\right)_{n} \frac{z^{n}}{n !}
$$

and

$$
\Phi_{p}\left(\vartheta_{2} ; \vartheta_{3} ; z\right)=\sum_{n=0}^{\infty} \frac{B_{p}\left(\vartheta_{2}+n, \vartheta_{3}-\vartheta_{2}\right)}{B\left(\vartheta_{2}, \vartheta_{3}-\vartheta_{2}\right)} \frac{z^{n}}{n !}
$$

where $p \geq 0$.

The integral representations of (1.7) and (1.8) respectively defined by

$$
\begin{aligned}
& F_{p}\left(\vartheta_{1}, \vartheta_{2} ; \vartheta_{3} ; z\right)=\frac{1}{B\left(\vartheta_{2}, \vartheta_{3}-\vartheta_{2}\right)} \\
& \times \int_{0}^{1} t^{\vartheta_{2}-1}(1-t)^{\vartheta_{3}-\vartheta_{2}-1}(1-z t)^{-\vartheta_{1}} \exp \left(\frac{-p}{t(1-t)}\right) d t \\
&\left(p \geq 0, \Re\left(\vartheta_{3}\right)>\Re\left(\vartheta_{2}\right)>0,|\arg (1-z)|<\pi\right)
\end{aligned}
$$

and

$$
\begin{gathered}
\Phi_{p}\left(\vartheta_{2}, \vartheta_{3} ; \gamma ; z\right)=\frac{1}{B\left(\vartheta_{2}, \vartheta_{3}-\vartheta_{2}\right)} \int_{0}^{1} t^{\vartheta_{2}-1}(1-t)^{\vartheta_{3}-\vartheta_{2}-1} \exp \left(z t-\frac{-p}{t(1-t)}\right) d t, \\
\left(p \geq 0, \Re\left(\vartheta_{3}\right)>\Re\left(\vartheta_{2}\right)>0\right) .
\end{gathered}
$$

The definition of extended Appell's function is (see [10])

$$
F_{1}\left(\vartheta_{1}, \vartheta_{2}, \vartheta_{3} ; \vartheta_{4} ; x, y ; p\right)=\sum_{n=0}^{\infty} \frac{B_{p}\left(\vartheta_{1}+m+n, \vartheta_{4}-\vartheta_{1}\right)}{\beta\left(\vartheta_{1}, \vartheta_{4}-\vartheta_{1}\right)}\left(\vartheta_{2}\right)_{m}\left(\vartheta_{3}\right)_{n} \frac{x^{m} y^{n}}{m ! n !}
$$

where $p \geq 0$ and its integral representation is

$$
F_{1}\left(\vartheta_{1}, \vartheta_{2}, \vartheta_{3} ; \vartheta_{4} ; x, y ; p\right)=\frac{1}{B\left(\vartheta_{1}, \vartheta_{4}-\vartheta_{1}\right)} \int_{0}^{1} t^{\vartheta_{1}-1}(1-t)^{\vartheta_{4}-\vartheta_{1}-1}(1-x t)^{-\vartheta_{2}}(1-y t)^{-\vartheta_{3}}
$$


A NEW EXTENSION OF EXTENDED CAPUTO FRACTIONAL...

$$
\begin{aligned}
& \times \exp \left(\frac{-p}{t(1-t)}\right) d t, \\
& \left(p \geq 0, \Re\left(\vartheta_{4}\right)>\Re\left(\vartheta_{1}\right)>0,|\arg (1-x)|<\pi,|\arg (1-y)|<\pi\right) .
\end{aligned}
$$

It is clear that when $p=0$, then the equations (1.7)-(1.12) reduce to the well known hypergeometric, confluent hypergeometric and Appell's series and their integral representation respectively (see [19]). Shadab et al. [5] introduced a new and modified extension of beta function very recently as:

$$
B_{p}^{\alpha}\left(\vartheta_{1}, \vartheta_{2}\right)=\int_{0}^{1} t^{\vartheta_{1}-1}(1-t)^{\vartheta_{2}-1} E_{\alpha}\left(-\frac{p}{t(1-t)}\right) d t
$$

where $\Re\left(\vartheta_{1}\right)>0, \Re\left(\vartheta_{2}\right)>0$ and $E_{\alpha}($.$) is Mittag-Leffler function defined by$

$$
E_{\omega}(z)=\sum_{n=0}^{\infty} \frac{z^{n}}{\Gamma(\omega n+1)} .
$$

Obviously, when $\omega=1$ then $B_{p}^{1}(x, y)=B_{p}(x, y)$ is the extended beta function (see[2]). Similarly, when when $\omega=1$ and $p=0$, then $B_{0}^{1}(x, y)=B_{0}(x, y)$ is the classical beta function.

The extended hypergeometric function and its integral representation due to [5] respectively defined byis

$$
\begin{aligned}
F_{p}^{\alpha}\left(\vartheta_{1}, \vartheta_{2} ; \vartheta_{3} ; z\right)={ }_{2} F_{1}\left(\vartheta_{1}, \vartheta_{2} ; \vartheta_{3} ; z ; p, \alpha\right) & =\sum_{n=0}^{\infty}\left(\vartheta_{1}\right)_{n} \frac{B_{p}^{\alpha}\left(\vartheta_{2}+n, \vartheta_{3}-\vartheta_{2}\right)}{B\left(\vartheta_{2}, \vartheta_{3}-\vartheta_{2}\right)} \frac{z^{n}}{n !} \\
& =\sum_{n=0}^{\infty}\left(\vartheta_{1}\right)_{n} \frac{B\left(\vartheta_{2}+n, \vartheta_{3}-\vartheta_{2} ; p, \alpha\right)}{B\left(\vartheta_{2}, \vartheta_{3}-\vartheta_{2}\right)} \frac{z^{n}}{n !}
\end{aligned}
$$

where $p, \alpha \geq 0, \vartheta_{1}, \vartheta_{2}, \vartheta_{3} \in \mathbb{C}$ and $|z|<1$.

$$
\begin{aligned}
F_{p}^{\alpha}\left(\vartheta_{1}, \vartheta_{2} ; \vartheta_{3} ; z\right) & =\frac{1}{\beta\left(\vartheta_{2} ; \vartheta_{3}-\vartheta_{2}\right)} \\
& \times \int_{0}^{1} t^{\vartheta_{2}-1}(1-t)^{\vartheta_{3}-\vartheta_{2}-1}(1-t z)^{-\vartheta_{1}} E_{\alpha}\left(-\frac{p}{t(1-t)}\right) d t,
\end{aligned}
$$

where $\Re(p)>0, \Re(\alpha)>0, \Re\left(\vartheta_{3}\right)>\Re\left(\vartheta_{2}\right)>0$. Obviously when $\alpha=1$, then the hypergeometric function (1.15) will reduce to the extended hypergeometric function (1.7) and similarly when $\alpha=1$ and $p=0$ then the hypergeometric function (1.15) will reduce to the hypergeometric function (1.1).

An interesting generalization of Appell hypergeometric function and its integral representation given in [17] as

$$
\begin{aligned}
F_{1, p}^{\alpha}\left(\vartheta_{1}, \vartheta_{2}, \vartheta_{3} ; \vartheta_{4} ; x, y\right) & =F_{1}\left(\vartheta_{1}, \vartheta_{2}, \vartheta_{3} ; \vartheta_{4} ; x, y ; p, \alpha\right) \\
& =\sum_{m, n=0}^{\infty}\left(\vartheta_{2}\right)_{m}\left(\vartheta_{3}\right)_{n} \frac{B_{p}^{\alpha}\left(\vartheta_{1}+m+n, \vartheta_{4}-\vartheta_{1}\right)}{B\left(\vartheta_{1}, \vartheta_{4}-\vartheta_{1}\right)} \frac{x^{m}}{m !} \frac{y^{n}}{n !}
\end{aligned}
$$




$$
=\sum_{m, n=0}^{\infty}\left(\vartheta_{2}\right)_{m}\left(\vartheta_{3}\right)_{n} \frac{B\left(\vartheta_{1}+m+n, \vartheta_{4}-\vartheta_{1} ; p, \alpha\right)}{B\left(\vartheta_{1}, \vartheta_{4}-\vartheta_{1}\right)} \frac{x^{m}}{m !} \frac{y^{n}}{n !}
$$

where $p, \alpha \geq 0, \vartheta_{1}, \vartheta_{2}, \vartheta_{3}, \vartheta_{4} \in \mathbb{C}$ and $|x|<1,|y|<1$.

$$
\begin{aligned}
F_{1}\left(\vartheta_{1}, \vartheta_{2}, \vartheta_{3} ; \vartheta_{4} ; x, y ; p, \alpha\right) & =\frac{1}{B\left(\vartheta_{1} ; \vartheta_{4}-\vartheta_{1}\right)} \int_{0}^{1} t^{\vartheta_{1}-1}(1-t)^{\vartheta_{4}-\vartheta_{1}-1}(1-t x)^{-\vartheta_{2}}(1-t y)^{-\vartheta_{3}} \\
& \times E_{\alpha}\left(-\frac{p}{t(1-t)}\right) d t
\end{aligned}
$$

where $\Re(p)>0, \Re(\alpha)>0, \Re\left(\vartheta_{4}\right)>\Re\left(\vartheta_{1}\right)>0,|\arg (1-x)|<\pi$ and $|\arg (1-y)|<\pi$. Recently, the researchers (see e. g., $[2-4,9-11,14,15])$ introduced various extension and generalization of various special functions. Also, they [17] give an extension fractional derivative operator of Riemann-Liouville as

$$
\begin{aligned}
\mathfrak{D}_{x ; p}^{\mu ; \alpha}\{f(x)\} & =\mathfrak{D}_{x}^{\mu}\{f(x) ; p, \alpha\} \\
& =\frac{1}{\Gamma(-\mu)} \int_{0}^{x} f(t)(x-t)^{-\mu-1} E_{\alpha}\left(-\frac{p x^{2}}{t(x-t)}\right) d t,
\end{aligned}
$$

where $\Re(\mu)>0, \Re(p)>0$ and $v \geq 0$. It is clear that, if $\alpha=1$, then definition 1.19 reduces to extended fractional derivative operator defined in [10]. Similarly, if $\alpha=1$ and $p=0$, then definition 1.19 reduces to classical fractional derivative operator defined in $[7]$.

\section{EXtension OF HYPERGEOMETRIC FUnCTIONS}

In this section, we define further extension of hypergeometric and Appell's hypergeometric functions.

Definition 2.1. The extension of extended hypergeometric function is defined as:

$$
\begin{aligned}
{ }_{2} F_{1, p}^{\alpha}\left(\vartheta_{1}, \vartheta_{2} ; \vartheta_{3} ; z\right) & ={ }_{2} F_{1}\left(\vartheta_{1}, \vartheta_{2} ; \vartheta_{3} ; z ; p ; \alpha\right) \\
& =\sum_{n=0}^{\infty} \frac{\left(\vartheta_{1}\right)_{n}\left(\vartheta_{2}\right)}{\left(\vartheta_{2}-m\right)_{n}} \frac{B_{p}^{\alpha}\left(\vartheta_{2}-m+n, \vartheta_{3}+m-\vartheta_{2}\right)}{B\left(\vartheta_{2}-m, \vartheta_{3}-\vartheta_{2}+m\right)} \frac{z^{n}}{n !},
\end{aligned}
$$

where $m-1<\Re\left(\vartheta_{2}-\vartheta_{3}\right)<m<\Re\left(\vartheta_{2}\right)>0, \Re(p)>0, \alpha>0$ and $|z|<1$.

Definition 2.2. The extension of Appell's hypergeometric function is defined as:

$$
\begin{aligned}
& F_{1, p}^{\alpha}\left(\vartheta_{1} ; \vartheta_{2}, \vartheta_{3} ; \vartheta_{3} ; z ; z\right)=F_{1}\left(\vartheta_{1} ; \vartheta_{2}, \vartheta_{3} ; \vartheta_{3} ; z ; z ; p ; \alpha\right) \\
= & \sum_{n, k=0}^{\infty} \frac{\left(\vartheta_{2}\right)_{n}\left(\vartheta_{3}\right)_{k}\left(\vartheta_{1}\right)_{n+k}}{\left(\vartheta_{1}-m\right)_{n+k}} \frac{z^{n}}{n !} \frac{z^{k}}{k !} \frac{B_{p}^{\alpha}\left(\vartheta_{1}+m+n+, \vartheta_{4}-\vartheta_{1}\right)}{B\left(\vartheta_{1}-m, \vartheta_{4}+m-\vartheta_{1}\right)},
\end{aligned}
$$

where $m-1<\Re\left(\vartheta_{1}-\vartheta_{4}\right)<m<\Re\left(\vartheta_{1}\right), \Re(p)>0, \alpha>0$.

The Integral representations of (2.1) and (2.2) are defined respectively as:

$$
{ }_{2} F_{1, p}^{\alpha}\left(\vartheta_{1}, \vartheta_{2} ; \vartheta_{3} ; z\right)=\frac{1}{B\left(\vartheta_{2}-m, \vartheta_{3}-\vartheta_{2}+m\right)} \int_{0}^{1} t^{\vartheta_{2}-m-1}(1-t)^{\vartheta_{3}-\vartheta_{2}+m-1}
$$


A NEW EXTENSION OF EXTENDED CAPUTO FRACTIONAL...

$$
\times E_{\alpha}\left(-\frac{p}{t(1-t)}\right){ }_{2} F_{1}\left(\vartheta_{1}, \vartheta_{2} ; \vartheta_{2}-m ; z t\right) d t
$$

and

$$
\begin{aligned}
F_{1}\left(\vartheta_{1}, \vartheta_{2}, \vartheta_{3} ; \vartheta_{4} ; z, z ; p, \alpha\right) & =\frac{1}{\beta\left(\vartheta_{1}-m, \vartheta_{4}-\vartheta_{1}+m\right)} \int_{0}^{1} t^{\vartheta_{1}-m-1}(1-t)^{\vartheta_{4}-\vartheta_{1}-m-1} \\
\times & E_{\alpha}\left(-\frac{p}{t(1-t)}\right) F_{1}\left(\vartheta_{1}, \vartheta_{2} ; \vartheta_{3} ; \vartheta_{1}-m ; z t ; z t\right) d t
\end{aligned}
$$

Remark 2.1. If we letting $\alpha=1$, then (2.1)-(2.4) reduces to the extended hypergeometric functions ${ }_{2} F_{1}$ and $F_{1}$ and their integral representations (see [6]) respectively.

\section{EXTENSION OF FRACTIONAL DERIVATIVE OPERATOR}

Recently, many papers (see $[1,8,12,16,21])$ introduced various extension and generalization of fractional calculus. In this section, we define a new extension of extended Caputo fractional derivative operator.

We recall the classical Caputo fractional derivative operator which is defined by

Definition 3.1. (see [7])

$$
\mathfrak{D}_{z}^{\mu}\{f(z)\}=\frac{1}{\Gamma(m-\mu)} \int_{0}^{z}(z-t)^{m-\mu-1} \frac{d^{m}}{d t^{m}} f(t) d t,
$$

where $m-1<\Re(\mu)<m$ where $m=1,2, \cdots$.

Recently Kiymaz et al. [6] introduced the extended Caputo fractional derivative operator as:

\section{Definition 3.2.}

$$
\mathfrak{D}_{z}^{\mu}\{f(z) ; p\}=\frac{1}{\Gamma(m-\mu)} \int_{0}^{z}(z-t)^{m-\mu-1} \exp \left(-\frac{p}{t(1-t)}\right) \frac{d^{m}}{d t^{m}} f(t) d t,
$$

where $m-1<\Re(\mu)<m$ where $m=1,2, \cdots$ and $\Re(p)>0$.

The extension of extended Caputo fractional derivative operator [18] defined by

\section{Definition 3.3.}

$$
\begin{aligned}
\mathfrak{D}_{z}^{\mu}\{f(z) ; p, v\} & =\mathfrak{D}_{z ; v}^{\mu ; p}\{f(z)\} \\
& =\sqrt{\frac{2 p z^{2}}{\pi}} \frac{1}{\Gamma(m-\mu)} \int_{0}^{z} t^{-\frac{1}{2}}(z-t)^{m-\mu-\frac{3}{2}} K_{v+\frac{1}{2}}\left(\frac{p z^{2}}{t(z-t)}\right) \frac{d^{m}}{d t^{m}} f(t) d t
\end{aligned}
$$

For the case $m-1<\Re(\mu)<m$ where $m=1,2, \cdots, \Re(p)>0 v \geq 0$.

In view of [17], we introduce a new extension Caputo fractional derivative operator and is defined as:

\section{Definition 3.4.}

$$
\mathfrak{D}_{z}^{\mu}\{f(z) ; p, \alpha\}=\mathfrak{D}_{z ; p}^{\mu ; \alpha}\{f(z)\}
$$




$$
=\frac{1}{\Gamma(m-\mu)} \int_{0}^{z}(z-t)^{m-\mu-1} E_{\alpha}\left(-\frac{p z^{2}}{t(z-t)}\right) \frac{d^{m}}{d t^{m}} f(t) d t
$$

for the case $m-1<\Re(\mu)<m$ where $m=1,2, \cdots, \Re(p)>0$.

Remark 3.1. Obviously if

(i) we letting $\alpha=1$, then definition 3.3 reduces to extended Caputo fractional derivative operator defined in 3.2 (see [6]).

(ii) we letting $\alpha=1$ and $p=0$, then definition 3.3 reduces to extended Caputo fractional derivative operator defined in 3.1 (see [7]).

Now, we prove some theorems involving the modified extension of fractional derivative operator.

Theorem 3.1. The following formula hold true,

$$
\mathfrak{D}_{z}^{\mu}\left\{z^{\eta} ; p, \alpha\right\}=\frac{\Gamma(\eta+1) B_{p}^{\alpha}(\eta-m+1, m-\mu)}{\Gamma(\eta-\mu+1) B(\eta-m+1, m-\mu)} z^{\eta-\mu}, \Re(\mu)>0,
$$

where $m-1<\Re(\mu)<m$ and $\Re(\mu)<\Re(\eta)$.

Proof. From (3.4), we have

$$
\begin{aligned}
\mathfrak{D}_{z}^{\mu}\left\{z^{\eta} ; p, \alpha\right\} & =\frac{1}{\Gamma(m-\mu)} \int_{0}^{z}(z-t)^{m-\mu-1} E_{\alpha}\left(-\frac{p z^{2}}{t(z-t)}\right) \frac{d^{m}}{d t^{m}} t^{\eta} d t \\
& =\frac{1}{\Gamma(m-\mu)}[\eta(\eta-1) \cdots(\eta-m+1)] \\
& \times \int_{0}^{z}(z-t)^{m-\mu-1} E_{\alpha}\left(-\frac{p z^{2}}{t(z-t)}\right) t^{\eta-m} d t \\
& =\frac{\Gamma(\eta+1)}{\Gamma(\eta-m+1) \Gamma(m-\mu)} \int_{0}^{z}(z-t)^{m-\mu} E_{\alpha}\left(-\frac{p z^{2}}{t(z-t)}\right) t^{\eta-m} d t
\end{aligned}
$$

Substituting $t=u z$ in (3.6), we have

$$
\mathfrak{D}_{z}^{\mu}\left\{z^{\eta} ; p, \alpha\right\}=\frac{\Gamma(\eta+1)}{\Gamma(\eta-m+1) \Gamma(m-\mu)} z^{\eta-\mu} \int_{0}^{z} u^{\eta-m}(1-u)^{m-\mu-1} E_{\alpha}\left(-\frac{p}{u(1-u)}\right) d u \text {. }
$$

By using the definition (1.13) to the above equation, we get

$$
\begin{aligned}
\mathfrak{D}_{z}^{\mu}\left\{z^{\eta} ; p, \alpha\right\} & =\frac{\Gamma(\eta+1)}{\Gamma(\eta-m+1) \Gamma(m-\mu)} z^{\eta-\mu} B_{p}^{\alpha}(\eta-m+1, m-\mu) \\
& =\frac{\Gamma(\eta+1) B_{p}^{\alpha}(\eta-m+1, m-\mu)}{\Gamma(\eta-\mu+1) B(\eta-m+1, m-\mu)} z^{\eta-\mu}
\end{aligned}
$$

which is the required result.

Theorem 3.2. Let $m-1<\Re(\mu)<m$ and suppose that the function $f(z)$ is analytic on the disk $|z|<r$ for some $r \in \mathbb{R}^{+}$and with its power series expansion given by $f(z)=$ $\sum_{n=0}^{\infty} a_{n} z^{n}$. Then

$$
\mathfrak{D}_{z}^{\mu}\{f(z) ; p, \alpha\}=\sum_{n=0}^{\infty} a_{n} \mathfrak{D}_{z}^{\mu}\left\{z^{n} ; p, \alpha\right\}
$$


Proof. Using the series expansion of the function $f(z)$ in (3.4) gives

$$
\mathfrak{D}_{z}^{\mu}\{f(z) ; p, \alpha\}=\frac{1}{\Gamma(m-\mu)} \int_{0}^{z}(z-t)^{m-\mu-1} E_{\alpha}\left(-\frac{p z^{2}}{t(z-t)}\right) \sum_{n=0}^{\infty} a_{n} \frac{d^{m}}{d t^{m}} t^{n} d t .
$$

As the series is uniformly convergent and the integrand is absolutely convergent, therefore interchanging the order of summation and integration gives

$$
\begin{aligned}
\mathfrak{D}_{z}^{\mu}\{f(z) ; p, \alpha\} & =\sum_{n=0}^{\infty} a_{n}\left\{\frac{1}{\Gamma(m-\mu)} \int_{0}^{z}(z-t)^{m-\mu-1} E_{\alpha}\left(-\frac{p z^{2}}{t(z-t)}\right) \frac{d^{m}}{d t^{m}} t^{n} d t\right\} \\
& =\sum_{n=0}^{\infty} a_{n} \mathfrak{D}_{z}^{\mu}\left\{z^{n} ; p, \alpha\right\}
\end{aligned}
$$

which is the required proof.

Theorem 3.3. Let $m-1<\Re(\mu)<m$ and suppose that the function $f(z)$ is analytic on the disk $|z|<r$ for some $r \in \mathbb{R}^{+}$and with its power series expansion given by $f(z)=$ $\sum_{n=0}^{\infty} a_{n} z^{n}$. Then

$$
\mathfrak{D}_{z}^{\mu}\left\{z^{\eta-1} f(z) ; p, \alpha\right\}=\frac{\Gamma(\eta) z^{\eta-\mu-1}}{\Gamma(\eta-\mu)} \sum_{n=0}^{\infty} a_{n} \frac{(\eta)_{n}}{(\eta-m)_{n}} \frac{B_{p}^{\alpha}(\eta-m+n, m-\mu)}{B(\eta-m, m-u)} z^{n}
$$

Proof. By applying Theorems (3.2) and (3.1), we have

$$
\begin{aligned}
\mathfrak{D}_{z}^{\mu}\left\{z^{\eta-1} f(z) ; p, \alpha\right\} & =\sum_{n=0}^{\infty} a_{n} \mathfrak{D}_{z}^{\mu}\left\{z^{\eta+n-1} ; p, \alpha\right\} \\
& =\frac{\Gamma(\eta) z^{\eta-\mu-1}}{\Gamma(\eta-\mu)} \sum_{n=0}^{\infty} a_{n} \frac{(\eta)_{n}}{(\eta-\mu)_{n}} \frac{B_{p}^{\alpha}(\eta-m+n, m-\mu)}{B(\eta-m+n, m-u)} z^{n} \\
& =\frac{\Gamma(\eta) z^{\eta-\mu-1}}{\Gamma(\eta-\mu)} \sum_{n=0}^{\infty} a_{n} \frac{(\eta)_{n}}{(\eta-m)_{n}} \frac{B_{p}^{\alpha}(\eta-m+n, m-\mu)}{B(\eta-m, m-u)} z^{n}
\end{aligned}
$$

which is the desired result.

Theorem 3.4. The following result holds true:

$$
\begin{gathered}
\mathfrak{D}_{z}^{\eta-\mu}\left\{z^{\eta-1}(1-z)^{-\beta} ; p, \alpha\right\}=\frac{\Gamma(\eta)}{\Gamma(\mu)} z^{\mu-1} \sum_{n=0}^{\infty} \frac{(\beta)_{n}(\eta)_{n}}{(\eta-m)_{n}} \frac{B_{p}^{\alpha}(\eta-m+n, m-\eta+\mu)}{B(\eta-m, \mu-\eta+m)} \frac{z^{n}}{n !}, \\
=\frac{\Gamma(\eta)}{\Gamma(\mu)} z^{\mu-1}{ }_{2} F_{1, p}^{\alpha}(\beta, \eta ; \mu ; z) .
\end{gathered}
$$

where $m-1<\Re(\eta-\mu)<m<\Re(\eta)>0, \Re(p)>0, \alpha>0$.

Proof. Using the power series of $(1-z)^{-\beta}$ and applying Theorem 3.1, we have

$$
\begin{aligned}
\mathfrak{D}_{z}^{\eta-\mu}\left\{z^{\eta-1}(1-z)^{-\beta} ; p, \alpha\right\} & =\mathfrak{D}_{z}^{\eta-\mu}\left\{z^{\eta-1} \sum_{n=0}^{\infty}(\beta)_{n} \frac{z^{n}}{n !} ; p, \alpha\right\} \\
& =\sum_{n=0}^{\infty} \frac{(\beta)_{n}}{n !} \mathfrak{D}_{z}^{\eta-\mu}\left\{z^{\eta+n-1} ; p, \alpha\right\}
\end{aligned}
$$




$$
\begin{aligned}
& =\sum_{n=0}^{\infty} \frac{(\beta)_{n}}{n !} \frac{\Gamma(\eta+n)}{\Gamma(\eta+n-m) \Gamma(m-\eta+\mu)} \\
& \times B_{p}^{\alpha}(\eta-m+n, m-\eta+\mu) z^{\mu+n-1} \\
& =\sum_{n=0}^{\infty} \frac{(\beta)_{n}}{n !} \frac{\Gamma(\eta+n)}{\Gamma(\mu+n)} \frac{B_{p}^{\alpha}(\eta-m+n, m-\eta+\mu)}{B(\eta-m+n, m-\eta+\mu)} z^{\mu+n-1} \\
& =\frac{\Gamma(\eta)}{\Gamma(\mu)} z^{\mu-1} \sum_{n=0}^{\infty} \frac{(\beta)_{n}(\eta)_{n}}{(\mu)_{n}} \frac{B_{p}^{\alpha}\left(\eta-m+n+\frac{1}{2}, m-\eta+\mu\right)}{B(\eta-m+n, m-\eta+\mu)} \frac{z^{n}}{n !} \\
& =\frac{\Gamma(\eta)}{\Gamma(\mu)} z^{\mu-1} \sum_{n=0}^{\infty} \frac{(\beta)_{n}(\eta)_{n}}{(\eta-m)_{n}} \frac{B_{p}^{\alpha}(\eta-m+n, m-\eta+\mu)}{B(\eta-m, \mu-\eta+m)} \frac{z^{n}}{n !},
\end{aligned}
$$

with the aid of (2.1), we get the required result.

Theorem 3.5. The following result holds true:

$$
\begin{aligned}
& \mathfrak{D}_{z}^{\eta-\mu}\left\{z^{\eta-1}(1-a z)^{-\alpha}(1-b z)^{-\beta} ; p, \alpha\right\} \\
= & \frac{\Gamma(\eta)}{\Gamma(\mu)} z^{\mu-1} \sum_{n, k=0}^{\infty} \frac{(\alpha)_{n}(\beta)_{k}(\eta)_{n+k}}{(\eta-m)_{n+k}} \frac{(a z)^{n}}{n !} \frac{(b z)^{k}}{k !} \frac{B_{p}^{\alpha}(\eta+m+n+k, \mu-\eta)}{B(\eta-m, m+\mu-\eta)} \\
= & \frac{\Gamma(\eta)}{\Gamma(\mu)} z^{\mu-1} F_{1}(\eta ; \alpha, \beta ; \mu ; a z ; b z ; p, \alpha)
\end{aligned}
$$

where $m-1<\Re(\eta-\mu)<m<\Re(\eta), \Re(p)>0, \alpha>0$.

Proof. To prove(3.10), we use the following power series expansion

$$
(1-a z)^{-\alpha}(1-b z)^{-\beta}=\sum_{n=0}^{\infty} \sum_{k=0}^{\infty}(\alpha)_{n}(\beta)_{k} \frac{(a z)^{n}}{n !} \frac{(b z)^{k}}{k !}
$$

Now, applying Theorem 3.4, we obtain

$$
\begin{aligned}
& \mathfrak{D}_{z}^{\eta-\mu}\left\{z^{\eta-1}(1-a z)^{-\alpha}(1-b z)^{-\beta} ; p, \alpha\right\} \\
& =\sum_{n=0}^{\infty} \sum_{k=0}^{\infty}(\alpha)_{n}(\beta)_{k} \frac{(a)^{n}}{n !} \frac{(b)^{k}}{k !} \mathfrak{D}_{z}^{\eta-\mu}\left\{z^{\eta+n+k-1} ; p, \alpha\right\}
\end{aligned}
$$

Using Theorem 3.1, we have

$$
\begin{aligned}
& \mathfrak{D}_{z}^{\eta-\mu}\left\{z^{\eta-1}(1-a z)^{-\alpha}(1-b z)^{-\beta} ; p, \alpha\right\} \\
& =\sum_{n=0}^{\infty} \sum_{k=0}^{\infty}(\alpha)_{n}(\beta)_{k} \frac{(a)^{n}}{n !} \frac{(b)^{k}}{k !} \frac{\Gamma(\eta+n+k) B_{p}^{\alpha}(\eta+m+n, \mu-\eta)}{\Gamma(m+\mu-\eta) \Gamma(\eta-m+n+k)} z^{\mu+n+k-1} \\
& =\frac{\Gamma(\eta)}{\Gamma(\mu)} z^{\mu-1} \sum_{n=0}^{\infty} \sum_{k=0}^{\infty} \frac{(\alpha)_{n}(\beta)_{k}(\eta)_{n+k}}{(\eta-m)_{n+k}} \frac{(a z)^{n}}{n !} \frac{(b z)^{k}}{k !} \frac{B_{p}^{\alpha}(\eta+m+n, \mu-\eta)}{B(\eta-m, m+\mu-\eta)}
\end{aligned}
$$

with the aid of (2.2), we get the required result. 


\section{Further Results of EXtended CAPUto FRACtional DERIVAtive operator}

In this section, we apply the extension of Caputo fractional derivative operator (3.4) to some known functions. Also, we investigate the Mellin transforms of the extension of Caputo fractional derivative operator.

Theorem 4.1. The following result holds true:

$$
\mathfrak{D}_{z}^{\mu}\left\{e^{z} ; p, \alpha\right\}=\frac{z^{m-\mu}}{\Gamma(m-\mu)} \sum_{n=0}^{\infty} \frac{z^{n}}{n !} B_{p}^{\alpha}(n+1, m-\mu)
$$

for all $z$.

Proof. Using the power series of $e^{z}$ and applying Theorems 3.2 and 3.1, we have

$$
\begin{aligned}
\mathfrak{D}_{z}^{\mu}\left\{e^{z} ; p, \alpha\right\} & =\sum_{n=0}^{\infty} \frac{z^{n}}{n !} \mathfrak{D}_{z}^{\mu}\left\{z^{n} ; p, \alpha\right\} \\
= & \sum_{n=m}^{\infty} \frac{\Gamma(n+1) B_{p}^{\alpha}(n-m+1, m-\mu)}{\Gamma(n-\mu+1) B(n-m+1, m-\mu)} \frac{z^{n-\mu}}{n !} \\
= & \sum_{n=0}^{\infty} \frac{\Gamma(n+m+1) B_{p}^{\alpha}(n+1, m-\mu)}{\Gamma(n+m-\mu+1) B(n+1, m-\mu)} \frac{z^{n+m-\mu}}{(n+m) !} \\
& =\frac{z^{m-\mu}}{\Gamma(m-\mu)} \sum_{n=0}^{\infty} \frac{z^{n}}{n !} B_{p}^{\alpha}(n+1, m-\mu) .
\end{aligned}
$$

Theorem 4.2. The following result holds true:

$$
\begin{aligned}
& \mathfrak{D}_{z}^{\mu}\left\{{ }_{2} F_{1}\left(\vartheta_{1}, \vartheta_{2} ; \vartheta_{3} ; z\right) ; p, \alpha\right\} \\
= & \frac{\left(\vartheta_{1}\right)_{m}\left(\vartheta_{2}\right)_{m}}{\left(\vartheta_{3}\right)_{m}} \frac{z^{m-\mu}}{\Gamma(1-\mu+m)} \sum_{n=0}^{\infty} \frac{\left(\vartheta_{1}+m\right)_{n}\left(\vartheta_{2}+m\right)_{n}}{\left(\vartheta_{3}+m\right)_{n}(1-\mu+m)_{n}} \frac{B_{p}^{\alpha}(n+1, m-\mu) z^{n}}{B(n+1, m-\mu)}
\end{aligned}
$$

for all $|z|<1$.

Proof. Using the power series of Gauss hypergeometric function ${ }_{2} F_{1}($.$) and applying The-$ orems 3.2 and 3.1, we have

$$
\begin{aligned}
& \mathfrak{D}_{z}^{\mu}\left\{{ }_{2} F_{1}\left(\vartheta_{1}, \vartheta_{2} ; \vartheta_{3} ; z\right) ; p, \alpha\right\} \\
= & \sum_{n=0}^{\infty} \frac{\left(\vartheta_{1}\right)_{n}\left(\vartheta_{2}\right)_{n}}{\left(\vartheta_{3}\right)_{n} n !} \mathfrak{D}_{z}^{\mu}\left\{z^{n} ; p, \alpha\right\} \\
= & \sum_{n=m}^{\infty} \frac{\left(\vartheta_{1}\right)_{n}\left(\vartheta_{2}\right)_{n}}{\left(\vartheta_{3}\right)_{n} n !} \frac{\Gamma(n+1) B_{p}^{\alpha}(n-m+1, m-\mu)}{\Gamma(n-\mu+1) B(n-m+1, m-\mu)} \frac{z^{n-\mu}}{n !} \\
= & \sum_{n=0}^{\infty} \frac{\left(\vartheta_{1}\right)_{n+m}\left(\vartheta_{2}\right)_{n+m}}{\left(\vartheta_{3}\right)_{n+m}(n+m) !} \frac{\Gamma(n+m+1) B_{p}^{\alpha}(n+1, m-\mu)}{\Gamma(n+m-\mu+1) B(n+1, m-\mu)} \frac{z^{n+m-\mu}}{(n+m) !}
\end{aligned}
$$


10

$$
=\frac{\left(\vartheta_{1}\right)_{m}\left(\vartheta_{2}\right)_{m}}{\left(\vartheta_{3}\right)_{m}} \frac{z^{m-\mu}}{\Gamma(1-\mu+m)} \sum_{n=0}^{\infty} \frac{\left(\vartheta_{1}+m\right)_{n}\left(\vartheta_{2}+m\right)_{n}}{\left(\vartheta_{3}+m\right)_{n}(1-\mu+m)_{n}} \frac{B_{p}^{\alpha}(n+1, m-\mu) z^{n}}{B(n+1, m-\mu)} \text {. }
$$

Theorem 4.3. The following result holds true:

$$
\mathfrak{D}_{z}^{\mu}\left[E_{\gamma, \delta}^{\vartheta}(z) ; p, \alpha\right]=\frac{z^{m-\mu}(\vartheta)_{m}}{\Gamma(m-\mu)} \sum_{n=0}^{\infty} \frac{(\vartheta)_{n}}{\Gamma(\gamma n+\gamma m+\delta)} B_{p}^{\alpha}(n+1, \mu-\eta) \frac{z^{n}}{n !},
$$

where $\gamma, \delta, \vartheta, \in \mathbb{C}, \Re(p)>0$, and $E_{\gamma, \delta}^{\vartheta}(z)$ is Mittag-Leffler function (see [13]) defined as:

$$
E_{\gamma, \delta}^{\vartheta}(z)=\sum_{n=0}^{\infty} \frac{(\vartheta)_{n}}{\Gamma(\gamma n+\delta)} \frac{z^{n}}{n !}
$$

Proof. Using (4.4) in (4.3), we have

$$
\mathfrak{D}_{z}^{\mu}\left[E_{\gamma, \delta}^{\vartheta}(z) ; p, \alpha\right]=\mathfrak{D}_{z}^{\mu}\left\{\sum_{n=0}^{\infty} \frac{(\vartheta)_{n}}{\Gamma(\gamma n+\delta)} \frac{z^{n}}{n !} ; p, \alpha\right\} .
$$

By Theorem 3.2, we have

$$
\mathfrak{D}_{z}^{\mu}\left\{E_{\gamma, \delta}^{\vartheta}(z) ; p, \alpha\right\}=\sum_{n=0}^{\infty} \frac{(\vartheta)_{n}}{\Gamma(\gamma n+\delta) n !}\left\{\mathfrak{D}_{z}^{\mu}\left\{z^{n} ; p, \alpha\right\}\right\} .
$$

Applying Theorem 3.1, we get

$$
\begin{aligned}
\mathfrak{D}_{z}^{\mu}\left\{E_{\gamma, \delta}^{\vartheta}(z) ; p, \alpha\right\} & =\sum_{n=m}^{\infty} \frac{(\vartheta)_{n}}{\Gamma(\gamma n+\delta)} \frac{\Gamma(n+1) B_{p}^{\alpha}(n-m+1, m-\mu)}{\Gamma(n-\mu+1) B(n-m+1, m-\mu)} \frac{z^{n-\mu}}{n !} \\
& =\sum_{n=0}^{\infty} \frac{(\vartheta)_{n+m}}{\Gamma(\gamma n+\gamma m+\delta)} \frac{\Gamma(n+m+1) B_{p}^{\alpha}(n+1, m-\mu)}{\Gamma(n+m-\mu+1) B(n+1, m-\mu)} \frac{z^{n+m-\mu}}{(n+m) !} \\
& =\frac{z^{m-\mu}(\vartheta)_{m}}{\Gamma(m-\mu)} \sum_{n=0}^{\infty} \frac{(\vartheta+m)_{n}}{\Gamma(\gamma n+\gamma m+\delta)} \frac{z^{n}}{n !} B_{p}^{\alpha}(n+1, m-\mu) .
\end{aligned}
$$

Theorem 4.4. The following result holds true:

$$
\begin{aligned}
\mathfrak{D}_{z}^{\mu}\left\{{ }_{m} \Psi_{n}\left[\begin{array}{c}
\left(\alpha_{i}, A_{i}\right)_{1, m} ; \\
\left(\beta_{j}, B_{j}\right)_{1, n} ;
\end{array}\right] ; p, \alpha\right\} & =\frac{z^{m-\mu}}{\Gamma(m-\mu)} \\
& \times \sum_{k=0}^{\infty} \frac{\prod_{i=1}^{m} \Gamma\left(\alpha_{i}+A_{i} k\right)}{\prod_{j=1}^{n} \Gamma\left(\beta_{j}+B_{j} k\right)} B_{p}^{\alpha}(n+1, m-\mu) \frac{z^{n}}{n !},
\end{aligned}
$$

where $\Re(p)>0, \alpha>0$ and ${ }_{m} \Psi_{n}(z)$ denotes Fox-Wright function defined by (see [7], pp. 56-58)

$$
{ }_{m} \Psi_{n}(z)={ }_{m} \Psi_{n}\left[\begin{array}{c}
\left(\alpha_{i}, A_{i}\right)_{1, m} ; \\
\left(\beta_{j}, B_{j}\right)_{1, n} ;
\end{array}\right]=\sum_{k=0}^{\infty} \frac{\prod_{i=1}^{m} \Gamma\left(\alpha_{i}+A_{i} k\right)}{\prod_{j=1}^{n} \Gamma\left(\beta_{j}+B_{j} k\right)} \frac{z^{k}}{k !} .
$$


Proof. Applying Theorem 3.1 and followed the same procedure used in Theorem 4.3, we get the desired result.

Theorem 4.5. The following Mellin transform formula holds true:

$$
\begin{aligned}
M\left\{\mathfrak{D}_{z ; p}^{\mu ; \alpha}\left(z^{\eta}\right) ; p \rightarrow r\right\} & =\frac{\pi}{\sin (\pi r)} \frac{\Gamma(\eta+1)}{\Gamma(1-r \alpha) \Gamma(m-\mu) \Gamma(\eta-m+1)} \\
& \times B(\eta-m+r+1, m-\mu+r) z^{\eta-\mu},
\end{aligned}
$$

where $\Re(\eta)>m-1, \Re(r)>0$.

Proof. Applying the Mellin transform on definition (3.3) and Theorem 3.1, we have

$$
\begin{aligned}
& M\left\{\mathfrak{D}_{z ; p}^{\mu ; \alpha}\left(z^{\eta}\right) ; p \rightarrow r\right\}=\int_{0}^{\infty} p^{r-1} \mathfrak{D}_{z ; p}^{\mu ; \alpha}\left(z^{\eta}\right) d p \\
= & \int_{0}^{\infty} p^{r-1}\left\{\frac{\Gamma(\eta+1)}{\Gamma(\eta-m+1) \Gamma(m-\mu)} \int_{0}^{z} t^{\eta-m}(1-u)^{m-\mu} E_{\alpha}\left(-\frac{p z^{2}}{t(z-t)}\right) d t\right\} d p \\
= & \frac{\Gamma(\eta+1)}{\Gamma(\eta-m+1) \Gamma(m-\mu)} z^{\eta-\mu} \int_{0}^{\infty} p^{r-1}\left\{\int_{0}^{1} u^{\eta-m}(1-u)^{m-\mu} E_{\alpha}\left(-\frac{p}{u(1-u)}\right) d u\right\} d p \\
= & \frac{\Gamma(\eta+1)}{\Gamma(\eta-m+1) \Gamma(m-\mu)} z^{\eta-\mu} \\
& \times \int_{0}^{1} u^{\eta-m}(1-u)^{m-\mu}\left(\int_{0}^{\infty} p^{r-1} E_{\alpha}\left(-\frac{p}{u(1-u)}\right) d p\right) d u .
\end{aligned}
$$

By substituting $v=\frac{p}{u(1-u)}(4.8)$ can be rewritten as

$$
\begin{aligned}
& M\left\{\mathfrak{D}_{z ; p}^{\mu ; \alpha}\left(z^{\eta}\right) ; p \rightarrow r\right\} \\
= & \frac{\Gamma(\eta+1)}{\Gamma(\eta-m+1) \Gamma(m-\mu)} z^{\eta-\mu} \\
& \left.\times \int_{0}^{1} u^{\eta+r-m}(1-u)^{m+r-\mu}\left(\int_{0}^{\infty} p^{r-1} E_{\alpha}(-v)\right) d v\right) d u
\end{aligned}
$$

By using the following formula,

$$
\int_{0}^{\infty} t^{s-1} E_{\alpha, \gamma}^{\delta}(-u t) d t=\frac{\Gamma(s) \Gamma(\delta-s)}{\Gamma(\delta) u^{s} \Gamma(\gamma-s \alpha)},
$$

for $\gamma=\delta=1$ and $u=1$ (4.9) becomes

$$
\begin{aligned}
& M\left\{\mathfrak{D}_{z ; p}^{\mu ; \alpha}\left(z^{\eta}\right) ; p \rightarrow r\right\} \\
= & \frac{\Gamma(\eta+1) \Gamma(r) \Gamma(1-r)}{\Gamma(1-r \alpha) \Gamma(\eta-m+1) \Gamma(m-\mu)} z^{\eta-\mu} \int_{0}^{1} u^{\eta+r-m}(1-u)^{m+r-\mu} d u \\
= & \frac{\Gamma(\eta+1) \Gamma(r) \Gamma(1-r)}{\Gamma(1-r \alpha) \Gamma(\eta-m+1) \Gamma(m-\mu)} z^{\eta-\mu} B(\eta+r-m+1,-\mu+r+m) \\
= & z^{\eta-\mu} \frac{\pi}{\sin (\pi r)} \frac{\Gamma(\eta+1)}{\Gamma(1-r \alpha) \Gamma(\eta-m+1) \Gamma(m-\mu)} B(\eta+r-m+1,-\mu+r+m) .
\end{aligned}
$$


Theorem 4.6. The following Mellin transform formula holds true:

$$
\begin{aligned}
& M\left\{\mathfrak{D}_{z ; p}^{\mu ; \alpha}\left((1-z)^{-\alpha}\right) ; p \rightarrow r\right\} \\
= & \frac{\pi}{\sin (\pi r)} \frac{(\alpha)_{m}}{\Gamma(1-r \alpha) \Gamma(m-\mu)} z^{m-\mu} \sum_{n=0}^{\infty}(\alpha+m)_{n} \frac{z^{n}}{n !} B(n+r+1, m-\mu+r),
\end{aligned}
$$

where $\Re(p)>0, \Re(r)>0$, and $|z|<1$.

Proof. Applying Theorem 4.5 with $\eta=n$ and using the power series extension of $(1-z)^{-\alpha}$, we can write

$$
\begin{aligned}
& M\left\{\mathfrak{D}_{z ; p}^{\mu ; \alpha}\left((1-z)^{-\alpha}\right) ; p \rightarrow r\right\}=\sum_{n=0}^{\infty} \frac{(\alpha)_{n}}{n !} M\left\{\mathfrak{D}_{z ; p}^{\mu ; \alpha}\left(z^{n}\right) ; p \rightarrow r\right\} \\
= & \frac{\pi}{\sin (\pi r) \Gamma(1-r \alpha) \Gamma(m-\mu)} \sum_{n=m}^{\infty} \frac{(\alpha)_{n} \Gamma(n+1)}{n ! \Gamma(n-m+1)} B(n-m+r+1, m-\mu+r) z^{n-\mu} \\
= & \frac{\pi}{\sin (\pi r) \Gamma(1-r \alpha) \Gamma(m-\mu)} z^{m-\mu} \sum_{n=0}^{\infty}(\alpha)_{n+m} \frac{z^{n}}{n !} B(n+r+1, m-\mu+r) \\
= & \frac{\pi}{\sin (\pi r)} \frac{(\alpha)_{m}}{\Gamma(1-r \alpha) \Gamma(m-\mu)} z^{m-\mu} \sum_{n=0}^{\infty}(\alpha+m)_{n} \frac{z^{n}}{n !} B(n+r+1, m-\mu+r),
\end{aligned}
$$

which is the required proof.

\section{Generating Relations}

In this section, we applying Theorems 3.4 and 3.5 and obtain generating relations for the extension of extended hypergeometric functions ${ }_{2} F_{1, p}^{\alpha}$ and $F_{1, p}^{\alpha}$.

Theorem 5.1. Assume that $m-1<\Re(\eta-\mu)<m<\Re(\eta)$ and $|z|<\min \{1,|1-t|\}$, then

$$
\sum_{n=0}^{\infty} \frac{(\lambda)_{n}}{n !}{ }_{2} F_{1, p}^{\alpha}(\lambda+n, \eta ; \mu ; z) t^{n}=(1-t)^{-\lambda}{ }_{2} F_{1, p}^{\alpha}\left(\lambda, \eta ; \mu \frac{z}{1-t}\right) .
$$

Proof. By considering the following series identity, we have

$$
[(1-z)-t]^{-\lambda}=(1-t)^{-\lambda}\left(1-\frac{z}{1-t}\right)^{-\lambda}
$$

Thus, the power series expansion yields

$$
\sum_{n=0}^{\infty} \frac{(\lambda)_{n}}{n !}(1-z)^{-\lambda}\left(\frac{t}{1-z}\right)^{n}=(1-t)^{-\lambda}\left[1-\frac{z}{1-t}\right]^{-\lambda}
$$

Multiplying both sides of (5.2) by $z^{\eta-1}$ and then applying the operator $\mathfrak{D}_{z ; p}^{\eta-\mu ; \alpha}$ on both sides, we have

$$
\mathfrak{D}_{z ; p}^{\eta-\mu ; \alpha}\left[\sum_{n=0}^{\infty} \frac{(\lambda)_{n}}{n !}(1-z)^{-\lambda}\left(\frac{t}{1-z}\right)^{n} z^{\eta-1}\right]=(1-t)^{-\lambda} \mathfrak{D}_{z ; p}^{\eta-\mu ; \alpha}\left[z^{\eta-1}\left(1-\frac{z}{1-t}\right)^{-\lambda}\right] .
$$


Interchanging the order of summation and the operator $\mathfrak{D}_{z ; p}^{\eta-\mu ; \alpha}$, we have

$$
\sum_{n=0}^{\infty} \frac{(\lambda)_{n}}{n !} \mathfrak{D}_{z ; p}^{\eta-\mu ; \alpha}\left[z^{\eta-1}(1-z)^{-\lambda-n}\right] t^{n}=(1-t)^{-\lambda} \mathfrak{D}_{z ; p}^{\eta-\mu ; \alpha}\left[z^{\eta-1}\left(1-\frac{z}{1-t}\right)^{-\lambda}\right] .
$$

Thus by applying Theorem 3.4, we obtain the required result.

Theorem 5.2. The following generating relation holds true:

$$
\sum_{n=0}^{\infty} \frac{(\lambda)_{n}}{n !}{ }_{2} F_{1 ; p}^{\alpha}(\delta-n, \eta ; \mu ; z) t^{n}=(1-t)^{-\beta} F_{1}\left(\delta, \lambda, \eta ; \mu ;-\frac{z t}{1-t} ; p, \alpha\right)
$$

where $|t|<\frac{1}{1+|t|}, m<\Re(\eta-\mu)<m<\Re(\eta)$.

Proof. Consider the series identity

$$
[1-(1-z) t]^{-\lambda}=(1-t)^{-\beta}\left[1+\frac{z t}{1-t}\right]^{-\lambda}
$$

Using the power series expansion to the left sides, we have

$$
\sum_{n=0}^{\infty} \frac{(\lambda)_{n}}{n !}(1-z)^{n} t^{n}=(1-t)^{-\lambda}\left[1-\frac{-z t}{1-t}\right]^{-\lambda}
$$

Multiplying both sides of (5.4) by $z^{\eta-1}(1-z)^{-\delta}$ and applying the operator $\mathfrak{D}_{z ; p}^{\eta-\mu ; \alpha}$ on both sides, we have

$\mathfrak{D}_{z ; p}^{\eta-\mu ; \alpha}\left[\sum_{n=0}^{\infty} \frac{(\lambda)_{n}}{n !} z^{\eta-1}(1-z)^{-\delta+n} t^{n}\right]=(1-t)^{-\lambda} \mathfrak{D}_{z ; p}^{\eta-\mu ; \alpha}\left[z^{\eta-1}(1-z)^{-\delta}\left(1-\frac{-z t}{1-t}\right)^{-\lambda}\right]$

where $\Re(\eta)>\Re(\mu)>0$ and $|z t|<|1-t|$, thus by Theorem 3.2, we have

$$
\sum_{n=0}^{\infty} \frac{(\lambda)_{n}}{n !} \mathfrak{D}_{z ; p}^{\eta-\mu ; \alpha}\left[z^{\eta-1}(1-z)^{-\delta+n}\right] t^{n}=(1-t)^{-\lambda} \mathfrak{D}_{z ; p}^{\eta-\mu ; \alpha}\left[z^{\eta-1}(1-z)^{-\delta}\left(1-\frac{-z t}{1-t}\right)^{-\lambda}\right] .
$$

Applying Theorem 3.5 on both sides, we get the desired result.

\section{Concluding Remarks}

In this paper, we established further extension of Caputo fractional derivative operator and obtained many results related to some known special functions and generating relations via special functions. We conclude that when $\alpha=1$ then all the results established in this paper will reduce to the results associated with extended Caputo fractional derivative operator defined by Kiymaz et. al. [6]. Similarly, if $\alpha=1$ and $p=0$ then all the results established in this paper will reduce to the results associated with classical Caputo fractional derivative operator (see [20]). 


\section{REFERENCES}

[1] D. Baleanu, P. Agarwal, R. K. Parmar, M. M. Alquarashi, S. Salahshour, Extension of the fractional derivative operator of the Riemann-Liouville, J. Nonlinear Sci. Appl., 10(2017), 2914-2924.

[2] M. A. Chaudhry, A. Qadir, M. Rafique, S. M. Zubair, Extension of Eulers beta function, J. Comput. Appl. Math. 78 (1997) 1932.

[3] M. A. Chaudhry, A. Qadir, H. M. Srivastava and R. B. Paris, Extended Hypergeometric and Confluent Hypergeometric functions, Appl. Math. Comput., 159 (2004) 589-602

[4] J. Choi, A. K. Rathie, R. K. Parmar, Extension of extended beta, hypergeometric and confluent hypergeometric functions, Honam Mathematical J. 36 (2014), No. 2, pp. 357-385.

[5] M. Shadab, S. Jabee and J. Choi, An extension of beta function and its application, Far East Journal of Mathematical Sciences, 103(2018), No. 1, pp 235-251.

[6] I. O. Kiymaz, A. Cetinkaya, P. Agarwal, An extension of Caputo fractional derivative operator and iyts application, J. Nonlinear Sci. Appl., 9 (2016), 3611-3621.

[7] A. A. Kilbas, H. M. Sarivastava, J. J. Trujillo, Theory and application of fractional differential equation, North-Holland Mathematics Studies, Elsevier Sciences B.V., Amsterdam, (2006).

[8] M. J. Luo, G. V. Milovanovic, P. Agarwal, Some results on the extended beta and extended hypergeometric functions, Appl. Math. Comput., 248 (2014), 631-651.

[9] S. Mubeen, G. Rahman, K. S. Nisar, J. Choi. M. Arshad, An extended beta function and its properties, Far East Journal of Mathematical Sciences, 102(2017), 1545-1557.

[10] M. A. Özerslan, E. Özergin, Some generating relations for extended hypergeometric functions via generalized fractional derivative operator, Mathematical and Computer Modelling, 52 (2010), 1825-1833.

[11] M. A. Özerslan, E. Özergin, Extension of gamma, beta and hypergeometric functions, J. Comput. Appl. Math. 235 (2011), 4601-4610.

[12] F. W. J. Olver, D. W. Lozier, R. F. Boisvert, C. W. Clark (eds.), NIST handbook of mathematical functions, With 1 CD-ROM (Windows, Macintosh and UNIX), U.S. Department of Commerce, National Institute of Standards and Technology, Washington, DC; Cambridge University Press, Cambridge, (2010).

[13] T.R. Prabhakar, A singular integral equation with a generalized MittagLeffler function in the kernel, Yokohama Math. J. 19, 715, (1971).

[14] R. K. Parmar, Some generating relations for generalized extended hypergeometric functions involving generalized fractional derivative operator, J. Concr. Appl. Math., 12 (2014), 217-228.

[15] R. K. Parmar, P. Chopra, R. B. Paris, On an extension of extended beta and hypergeometric functions, arXiv:1502.06200 [math.CA], 22, 2015. [to appear in J. Classical Anal.] 
[16] G. Rahman, S. Mubeen, K. S. Nisar, J. Choi, Extended special functions and fractional integral operator via an extended Beta function, Submitted.

[17] G. Rahman, K. S. Nisar, Z. Tomovski, A new extension of the Riemann-Liouville fractional derivative operator, Submitted.

[18] G. Rahman, S. Mubeen, M. Arshad, FOn Extended Caputo Fractional Derivative Operator, DOI: 10.20944/preprints201712.0195.v1.

[19] E. D. Rainville, Special functions, The Macmillan Company, New York, 1960.

[20] S. G. Samko, A. A. Kilbas, O. I. Marichev, Fractional integrals and derivatives, Theory and applications, Edited and with a foreword by S. M. Nikolski ${ }^{\sim}$, Translated from the 1987 Russian original, Revised by the authors, Gordon and Breach Science Publishers, Yverdon, (1993).

[21] H. M. Srivastava, R. K. Parmar, P. Chopra, A class of extended fractional derivative operators and associated generating relations involving hypergeometric functions, Axioms, 1 (2012), 238-258.

Gauhar Rahman: Department of Mathematics, International Islamic University, IsLAMABad, PAKISTAN

E-mail address: gauhar55uom@gmail.com

Kottakkaran Sooppy Nisar: Department of Mathematics, College of Arts and ScienceWadi Aldawaser, 11991, Prince Sattam bin Abdulaziz University, Alkharu, Kingdom of SAUdi Arabia

E-mail address: n.sooppy@psau.edu.sa; ksnisar1@gmail.com

Shahid Mubeen: Department of Mathematics, University of Sargodha, Sargodha, PakISTAN

E-mail address: smjhanda@gmail.com 